\title{
Vanishing Carrier-Envelope-Phase-Sensitive Response in Optical-Field Photoemission from Plasmonic Nanoantennas
}

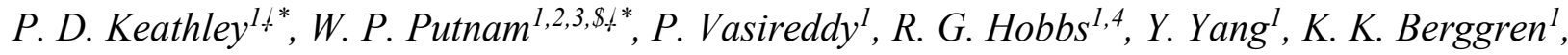 \\ and F. X. Kärtner ${ }^{1,2,5}$ \\ ${ }^{1}$ Research Laboratory of Electronics, Massachusetts Institute of Technology, 77 Massachusetts Ave., Cambridge, MA 02139, USA \\ ${ }^{2}$ Department of Physics and Center for Ultrafast Imaging, University of Hamburg, Luruper Chaussee 149, 22761 Hamburg, Germany \\ ${ }^{3}$ Northrop Grumman Corporation, NG Next, 1 Space Park Blvd., Redondo Beach, CA 90278, USA \\ ${ }^{4}$ Centre for Research on Adaptive Nanostructures and Nanodevices (CRANN), Advanced Materials and Bio-Engineering Research Centre \\ (AMBER), and School of Chemistry, Trinity College Dublin, Dublin 2, Ireland \\ ${ }^{5}$ Center for Free-Electron Laser Science and Deutsches Elektronen-Synchrotron (DESY), Notkestraße 85, 22607 Hamburg, Germany \\ ${ }^{\$}$ Current address: Department of Electrical and Computer Engineering, University of California, Davis, 1 Shields Ave., Davis, CA 95616, USA \\ *These authors contributed equally to this work. \\ *Corresponding authors: pdkeat2@mit.edu and bputnam@ucdavis.edu
}

At the surfaces of nanostructures, enhanced electric fields can drive optical-field photoemission and thereby generate and control electrical currents at frequencies exceeding $100 \mathrm{THz}^{1-11}$. A hallmark of such optical-field photoemission is sensitivity of the total emitted current to the carrierenvelope phase $(\mathrm{CEP})^{1-3,7,11-17}$. Here we examine CEP-sensitive photoemission from plasmonic gold nanoantennas excited with few-cycle optical pulses. At a critical pulse energy, which we call a vanishing point, we observe a pronounced dip in the magnitude of the CEP-sensitive photocurrent accompanied by a sudden shift of $\pi$-radians in the photocurrent phase. Analysis shows that this vanishing behavior arises due to competition between sub-optical-cycle electron emission events from neighboring optical half-cycles, and that both the dip and phase shift are highly sensitive to the precise shape of the driving optical waveform at the surface of the emitter. As the mechanisms underlying the dip and phase shift are a general consequence of nonlinear, field-driven photoemission, they may be used to probe sub-optical-cycle emission processes from solid-state emitters, atoms, and molecules. Improved understanding of these CEP-sensitive photocurrent features will be critical to the development of optical-field-driven photocathodes for time-domain metrology and microscopy applications demanding attosecond temporal and nanometer spatial resolution. 

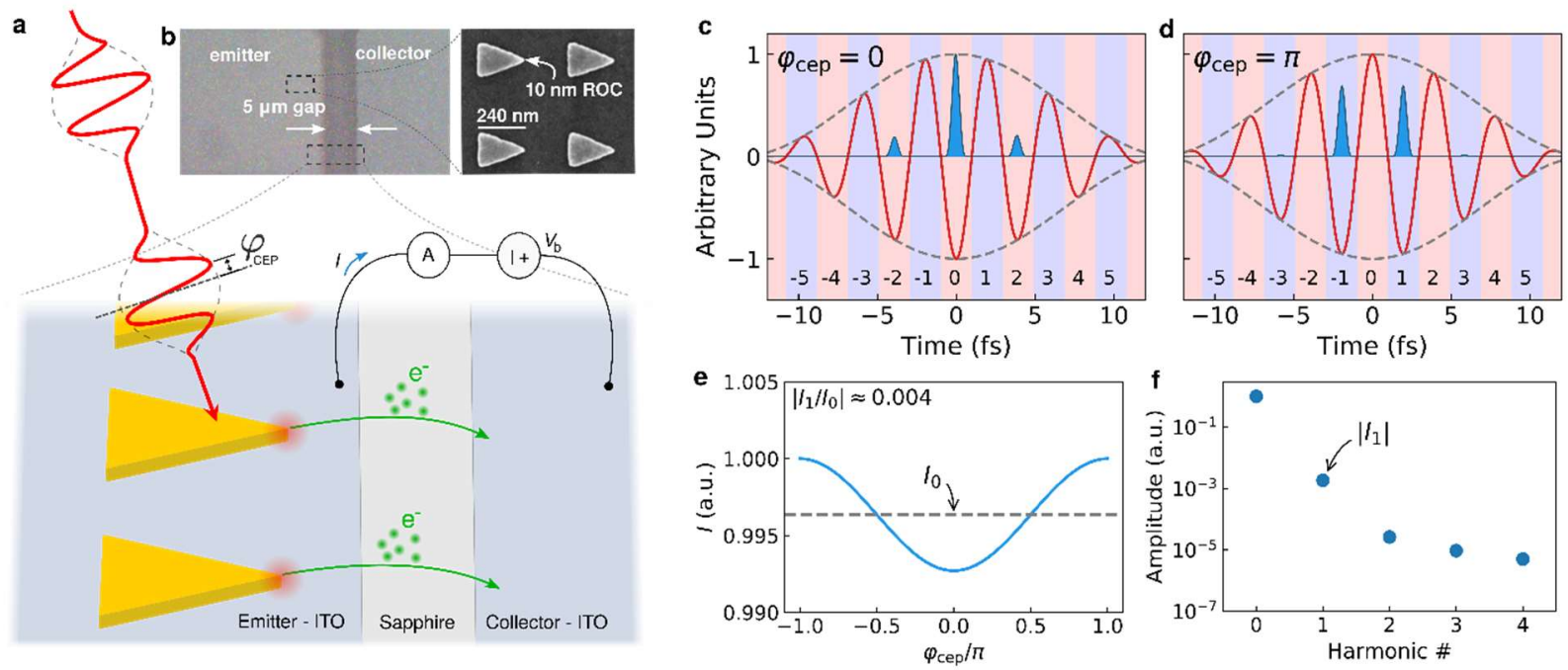

Fig. 1 Experimental setup and illustration of CEP-sensitivity. a. Illustration showing optical excitation and charge extraction from the nanoantenna array in the experimental setup. Photocurrent is pulled by a bias voltage, $\mathrm{V}_{\mathrm{D}}=30 \mathrm{~V}$, from the nanoantenna array into an isolated collector electrode where it is then detected using a transimpedance amplifier. b. Optical microscope image of emitter array and collector region (left) and scanning electron micrograph of triangular gold nanoantennas used in the experiment (right). c. Simulated waveform (red curve) along with calculated electron emission rate (filled blue curves) as a function of time with $\varphi_{\text {cep }}=0$. Half-cycle numbers are labeled across the bottom. Note the half-cycle regions are determined relative to the center of the pulse intensity envelope, not the field waveform (i.e. they do not depend on $\left.\varphi_{\text {cep}}\right)$. d. Same as $\mathbf{c}$, but with $\varphi_{\text {cep }}=\pi$. e. Plot of the total emitted current as a function of $\varphi_{\text {cep }}$. The average total current $I_{0}$ is labeled. The magnitude of the CEP-sensitivity was found to be $\left|\frac{I_{1}}{I_{0}}\right|=0.004$. f. Magnitudes of the harmonic components of the CEP-sensitive current $I_{n}$ for $n=0$ to $n=4$.

Our experimental setup is shown in Fig. 1a. We illuminate an array of triangular, gold nanoantennas (nano-triangles) that sit on a conductive ITO layer with pulses of near-infrared light having a central wavelength of of $\approx 1177 \mathrm{~nm}$ and a duration of $10 \mathrm{fs}$, that is 2.5 optical cycles, full-width-at-half-maximum (FWHM). The incident laser pulses are polarized along the altitudes of the triangular nanoantennas and drive electron emission. Field-enhancement factors of $\approx 30 \times$ are achieved near the apices of the triangles due to both plasmonic resonance as well as geometric

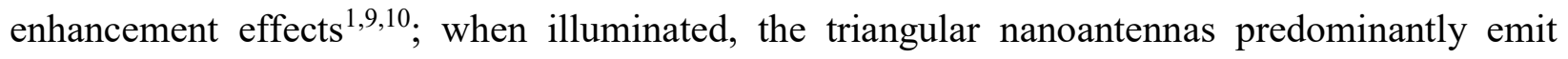
electrons from their apices due to these localized, enhanced fields ${ }^{18}$. Following emission, a DC bias voltage sweeps the electrons from the antennas, through the interstitial air, and across an 
insulating gap of roughly $5 \mu \mathrm{m}$ to a second ITO pad serving as a collector. Such a short gap size reduces the likelihood of electron capture and removes the need for the samples to be housed in vacuum. Optical and scanning-electron micrographs of the emitter electrode, gap, collector electrode, and emitter structures are shown Fig. $1 \mathrm{~b}$.

With sufficiently high fields at the emitter apices, the tips are driven into the optical-field photoemission regime where the emission can be approximated by a quasi-static tunneling rate $^{1,8,19-21}$. In Figs. 1c,d, we show the emitted electron current, as calculated by a quasi-static Fowler-Nordheim (FN) emission model, which has proven effective at predicting optical-fielddriven photocurrents from gold nanotips and nanoantennas ${ }^{1,2,4,11,21}$ (see Methods for further details). For the calculation we use a $10 \mathrm{fs}$ laser pulse with a central wavelength of $\lambda=1177 \mathrm{~nm}$ and a peak field of $F_{0}=15 \mathrm{GV} / \mathrm{m}$. The work function of the nanoantennas was taken to be $\phi=$ $5.1 \mathrm{eV}$ (we use this value of the work function for the remainder of the paper). For FN tunneling emission, current is emitted in sub-cycle bursts during half-cycles where the field is negative. Emission is suppressed during half-cycles where the field is positive as the surface electrons experience a force that drives them further into the emitter. As it will be convenient for later analysis, in Figs. 1c,d we have shaded and numbered half-cycle regions of the pulse (even halfcycles are shaded light red, and odd ones are shaded light blue). From Fig. 1c,d, it is apparent that the CEP of the driving laser pulse, $\varphi_{\text {cep }}$, has a strong effect on the emission. As $\varphi_{\text {cep }}$ is changed from 0 (Fig. 1c) to $\pi$ (Fig. 1d), the total emitted charge per pulse switches from being dominated by even half-cycle contributions (Fig. 1c) to being dominated by odd half-cycle contributions (Fig. 1d). (Note that the half-cycle regions are defined relative to the center of the intensity envelope, not the underlying field waveform, and thus do not move when the CEP is shifted.)

To better quantify the impact of $\varphi_{\text {cep }}$ on the measured photocurrent, we need to define the 
key observables for both our measurements and subsequent analysis. In Fig. 1e we plot the integrated total photoemission current $I_{\text {tot }}\left(\varphi_{\text {cep }}\right)$ (see Methods for calculation details). Note that $I_{\text {tot }}$ has been normalized in the plot. Using harmonic analysis, the integrated total photoemission current can be written as $I_{\text {tot }}\left(\varphi_{\text {cep }}\right)=\sum_{n}\left|I_{n}\right| \cos \left(n \varphi_{\text {cep }}+\angle I_{n}\right)$ where $I_{n}$ is the complex amplitude of the $n^{\text {th }}$ harmonic of the CEP-dependent total photoemission current. In Fig. $1 \mathrm{f}$ we plot $\left|I_{n}\right|$ for $n=0$ to $n=4$ on a logarithmic scale. We note that $I_{\text {tot }}\left(\varphi_{\text {cep }}\right)$ is dominated by the CEP-independent average total photocurrent $I_{0}$ and the first harmonic, $I_{1}$, i.e. the fundamental CEP-dependent sinusoidal component of the photocurrent. We also define the complex ratio $I_{1} / I_{0}$ as the CEP-sensitivity.

For experimental characterization, we phase-stabilized our optical source with a fixed carrier-envelope offset frequency $f_{\text {ceo }}=100 \mathrm{~Hz}$ such that $\varphi_{\text {cep }}=2 \pi f_{\text {ceo }} t$, and used lock-in detection to characterize $I_{1}$ (see Methods and Ref. ${ }^{1}$ for further details). We then monitored $I_{1}$ while scanning through a range of incident pulse energies; the experimental results are plotted in Fig. 2 (orange circles). Intuitively, it would seem that at higher intensities, when the overall photoemission current is larger and when we are deeper into the optical-field photoemission regime, that we would observe a larger CEP-sensitive photocurrent. However, we find a striking behavior in the experimental data where at a critical pulse energy of $\approx 0.1 \mathrm{~nJ}$ we observe a dramatic dip in $\left|I_{1}\right|$ and a corresponding phase-shift of $\pi$-radians in $\angle I_{1}$. We refer to this critical pulse energy as a vanishing point; the behavior at this vanishing point was measured multiple times at various locations on the sample surface over several days of measurement (see Supplementary Information Fig. 2 and Supplementary Information Section III). We note that the observed dip in amplitude and the phase shift are reminiscent of the characteristics of an antiresonance. (The antiresonance analogy holds in some respects beyond just the appearance of 
the data; however, the observed dip and phase shift appear with changes in driving pulse energy, not frequency, on the horizontal axis and are not representative of an antiresonance in a strict sense.)
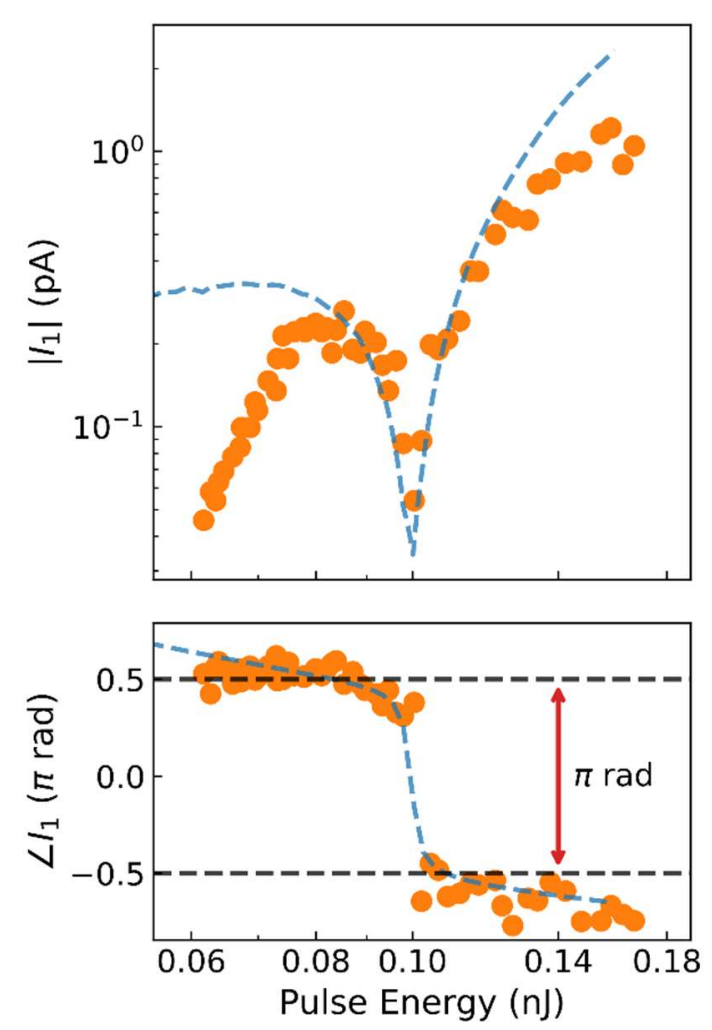

Fig. 2 Measurement of $\left|I_{1}\right|$ and $\angle I_{1}$ as a function of the incident pulse energy (orange circles). Model results (blue dashed curve) are overlaid on top of the experimental data. At a pulse energy of $\approx 0.1 \mathrm{~nJ}$, a simultaneous dip in $\left|I_{1}\right|$ and phase jump of $\pi$-radians in $\angle I_{1}$ are observed. (A pulse energy of $\approx 0.1 \mathrm{~nJ}$ corresponds to a peak enhanced field of $\approx 35 \mathrm{GV} / \mathrm{m}$, and peak enhanced intensity of $\approx 1.6 \times 10^{14} \mathrm{~W} / \mathrm{cm}^{2}$.) Our quasi-static model provides an excellent fit to the experimental data when using a field enhancement factor of $32.6 \times$, a resonant wavelength of $\lambda_{\text {res }} \approx 1105 \mathrm{~nm}$, and a damping time of $\tau=6.8 \mathrm{fs}$, which match well with values obtained from separate measurements.

Overlaid on our experimental results in Fig. 2 are the predicted CEP-dependent behaviors from our quasi-static tunneling model (shown as a blue dashed line). Note that for the model results in Fig. 2, we have accounted for the effects of the plasmonic resonance of the nanoparticles using a damped harmonic oscillator model, and spatial averaging over the beam spot (see Methods for calculation details). From Fig. 2, we see that the quasi-static model shows excellent agreement with the experimental data in both magnitude and phase, especially at high intensities where the 
photoemission is deep in the optical-field-emission regime. When modeling the CEP-sensitive photocurrent response, we used a plasmonic resonance wavelength of $\lambda_{\text {res }} \approx 1105 \mathrm{~nm}$ and a field enhancement of $32.6 \times$; these values were extracted from extinction and photocurrent scaling measurements on the nanoantenna arrays (see Methods and Supplementary Information Sections IV-VI). Extinction measurements indicated a plasmonic damping time of $\tau=6.4-6.9 \mathrm{fs}$; in our model, we used $\tau=6.8$ fs. We should note that changes in the modeled damping time of just 100 as lead to visually apparent changes in the shape of $\left|I_{1}\right|$ and $\angle I_{1}$ as a function of intensity near the vanishing point (see Supplementary Information Fig. 5). The discrepancy between our model results and the experimental data is most pronounced at low intensities; this is not unexpected as at these intensities the emission approaches the multiphoton regime, and thus the quasi-static FN model provides a poorer estimate of the total current response.

The simulation results shown in Fig. 2 accurately account for the experimental behaviors and give us confidence that our modeling of optical-field photoemission as a quasi-static tunneling process is reasonable. However, the simulation results provide little insight into the physical origin of the dip and phase shift in the CEP-dependent photocurrent. To get a sense for these origins, we now turn to a simplified version of the model presented in Fig. 2. In this simplified model, we ignored the filtering of the incident pulse by the plasmonic response of the nanoantennas as well as the focal spot averaging. We simply used a transform-limited pulse with similar duration (10 fs FWHM) and central wavelength $(1177 \mathrm{~nm})$ to that used in the experiment and considered emission from a single nanoantenna. The results are presented in Fig. 3.

In Fig. 3 we examine properties of the electron emission as a function of intensity assuming emission only from the red and blue shaded regions of the optical pulse shown in the top-left inset of Figs. 3a,b,c. The top panels of Figs. 3a,b,c show $\left|I_{1}\right|$, and the bottom panels show $\angle I_{1}$ as a 

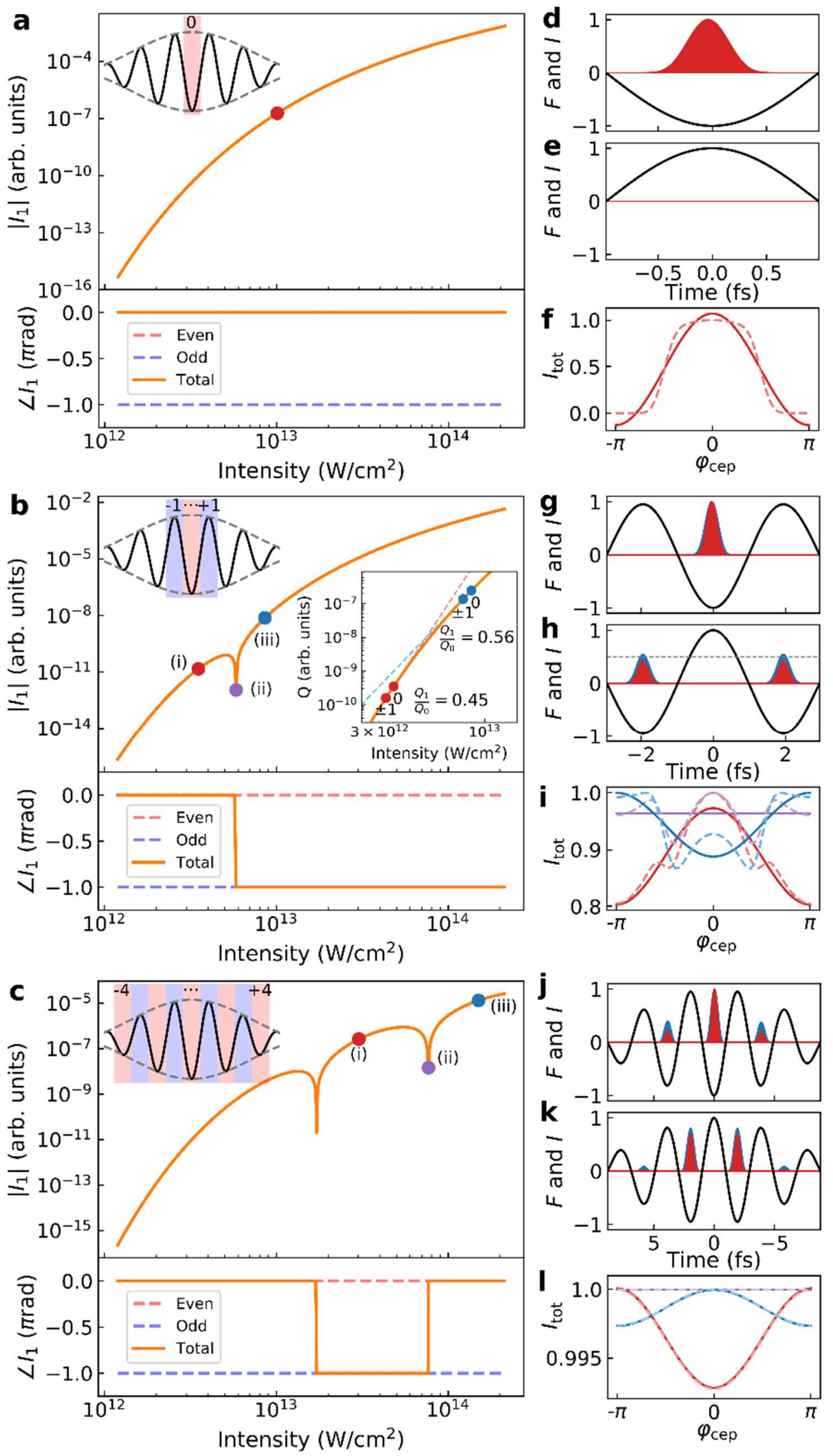
Fig. 3 Study of vanishing points in CEP-sensitive photocurrent excited by a transform-limited pulse. ac. Plot of $\left|I_{1}\right|$ (top panel) and $\angle I_{1}$ (bottom panel) as a function of peak intensity when only considering emission from the shaded region shown in the top-left inset (the driving pulse is a transform-limited, $\cos ^{2}$-shaped pulse with FWHM of $10 \mathrm{fs}$ and central wavelength of $1177 \mathrm{~nm}$ ). The light red and light blue dashed lines in the bottom panels indicate the phase response when considering only the shaded even half-cycle contributions and only the shaded odd half-cycle contributions respectively. As opticalfield emission is only relevant for Keldysh parameters ${ }^{22} \gamma$ on the order of unity or below, we only simulate intensities above $10^{12} \mathrm{~W} / \mathrm{cm}^{2}$, which corresponds to Keldysh parameters $\gamma<4.5$. In $\mathbf{b}$, the lower-right inset shows the relative charge emission $Q$ per half-cycle as a function of peak intensity (orange curve). The charge from half-cycles $0, \pm 1$ are also shown at intensities (i) and (iii) (red and blue markers). d. Plot of the normalized emitted photocurrent as a function of time (shaded red curve) for half-cycle 0 at the intensity shown by the red marker in a with $\varphi_{\text {cep }}=0$. The black curve is the electric field waveform. e. Same as d, but for $\varphi_{\text {cep }}=\pi$. f. Normalized total photocurrent $I_{\text {tot }}$ as a function of $\varphi_{\text {cep }}$ considering only contributions from shaded half-cycle in $\mathbf{a}$. The solid curve shows only the $I_{0}$ and $I_{1}$ contributions together for reference. $\mathbf{g}-\mathbf{i}$ and $\mathbf{j}-\mathbf{l}$. Same as $\mathbf{d}-\mathbf{f}$ but considering emission arising only from the shaded half-cycles in $\mathbf{b}$ and $\mathbf{c}$ respectively. The red, blue, and purple curves correspond to three intensity values indicated by markers in $\mathbf{b}$ and $\mathbf{c}$ : (i) just before the selected vanishing point, (ii) at the critical intensity of the selected vanishing point, and (iii) just after the selected vanishing point. Note that in $\mathbf{h}$, it is clear that the \pm 1 half-cycles dominate after exceeding the dashed line indicating half the peak current from half-cycle 0 .

function of optical intensity. Note that the shaded regions encompass an increasing number of half-cycles moving from the center of the pulse out to the wings: in Fig. 3a only one half-cycle is shaded (we consider emission from only the center half-cycle, i.e. half-cycle 0), in Fig. $3 b$ three are shaded (we consider emission from half-cycles 0 and \pm 1 ), and in Fig. $3 c$ nine are shaded (we consider emission from half-cycles -4 to +4$)$. As with Figs. 1c,d, we shade even half-cycles in light red and odd half-cycles in light blue. Additionally, in each plot of $\angle I_{1}$, there is a light red dashed line "even" showing the phase response of the sum of the even half-cycle contributions to $I_{1}$ (i.e. contributions from half-cycles $0, \pm 2, \pm 4, \ldots$ ), and a light blue dashed line called "odd" showing the phase response of the sum of the odd half-cycle contributions to $I_{1}$ (i.e. contributions from half-cycles $\pm 1, \pm 3, \pm 5, \ldots)$. Note the sum of the even half-cycle contributions always peaks at $\varphi_{\text {cep }}=0$, while the sum of the odd half-cycle contributions always peaks at $\varphi_{\text {cep }}= \pm \pi$.

In Figs. 3d,e,f, we analyze the emission from half-cycle 0 at the intensity labeled by the 
red dot in Fig. 3a. Fig. 3d shows the normalized emitted photocurrent (red) and half-cycle 0 of the driving electric field waveform (black curve) for $\varphi_{\text {cep }}=0$. Fig. 3e similarly shows the normalized emitted photocurrent and driving electric field for $\varphi_{\text {cep }}=\pi$. Note that since tunneling requires a negative field, a large photocurrent pulse appears for $\varphi_{\text {cep }}=0$, while no photocurrent appears for $\varphi_{\text {cep }}=\pi$. In Fig. 3f, we plot $I_{\text {tot }}\left(\varphi_{\text {cep }}\right)$ with a red dashed line, and we plot $I_{0}+$ $\left|I_{1}\right| \cos \left(\varphi_{\text {cep }}+\angle I_{1}\right)$ with a red solid line. From Fig. 3f, we see that the emitted current is maximized at $\varphi_{\text {cep }}=0$ and minimized at $\varphi_{\text {cep }}=\pi$ (where there is no emission).

In Figs. 3g,h,i, we similarly analyze emission from half-cycles 0 and \pm 1 . However, in Fig. 3g,h we show the photocurrent emission at two intensities: in red, we show the photocurrent from the intensity labeled (i), and indicated with a red dot in Fig. 3b, and in blue, we display the photocurrent at the intensity labeled (iii) and shown with a blue dot in Fig. 3b. Fig. 3g shows the normalized emitted photocurrents (red and blue) and half-cycles 0 and \pm 1 of the driving electric field waveform (black) for $\varphi_{\text {cep }}=0$. In Fig. $3 \mathrm{~g}$ the photocurrents plotted in red and blue are multiplied by normalization constant $C_{\text {red }}$ and $C_{\text {blue }}$ respectively, such that their maximum values are 1. Fig. $3 \mathrm{~h}$ shows the emitted photocurrents (red and blue) and half-cycles 0 and \pm 1 of the driving electric field waveform (black) for $\varphi_{\text {cep }}=\pi$. Additionally, in Fig. $3 \mathrm{~h}$ the same normalization constants as used in Fig. $3 \mathrm{~g}, C_{\text {red }}$ and $C_{\text {blue }}$, are used, i.e. the photocurrents are normalized relative to those plotted in Fig. 3g.

Consider the peak values of the photocurrents in Fig. 3h. The dashed black line in Fig. 3h denotes 0.5 . The photocurrents plotted in red lie just below this line, and those in blue lie just above it. Therefore, if we sum the photocurrents in red from the \pm 1 half-cycles, we expect that this total current will be smaller than that from half-cycle 0 . Likewise, if we sum the photocurrents in blue from the \pm 1 half-cycles, we expect that this total current will be larger than that from half- 
cycle 0 . In other words, we expect that at intensity (i), $I_{\text {tot }}\left(\varphi_{\text {cep }}\right)$ will be maximized at $\varphi_{\text {cep }}=0$ while at intensity (iii), $I_{\text {tot }}\left(\varphi_{\text {cep }}\right)$ will be maximized at $\varphi_{\text {cep }}=\pi$. Moreover, between intensity (i) and (iii), we anticipate an intensity where the sum of the photocurrents from the \pm 1 half-cycles and the photocurrents from half-cycle 0 are about equal. Here, we expect $I_{\text {tot }}\left(\varphi_{\text {cep }}=0\right) \approx$ $I_{\text {tot }}\left(\varphi_{\text {cep }}=\pi\right)$, and we therefore expect that the first harmonic component of $I_{\text {tot }}\left(\varphi_{\text {cep }}\right)$ will approximately vanish (i.e. that $I_{1} \approx 0$ ). This intensity is the vanishing point, and we label it as intensity (ii) and mark it with a purple circle in Fig. 3b.

In Fig. 3i, we plot $I_{\text {tot }}\left(\varphi_{\text {cep }}\right)$ for intensities (i), (ii), and (iii) with red, purple, and blue dashed lines respectively, and we overlay plots of $I_{0}+\left|I_{1}\right| \cos \left(\varphi_{\text {cep }}+\angle I_{1}\right)$ for these three intensities with solid lines of the same color. Note that in Fig. 3i we observe the exact behaviors described in the preceding paragraph: moving from intensity (i) to (ii) to (iii), $I_{\text {tot }}\left(\varphi_{\text {cep }}\right)$ transitions from being peaked at $\varphi_{\text {cep }}=0$, to having a minimal first harmonic, to being peaked at $\varphi_{\text {cep }}=\pi$. We should also note that these behaviors are consequences of the nonlinear character of the fielddriven photoemission. In the inset of Fig. 3b, we display the emitted charge, i.e. the integrated photocurrent, from an individual half-cycle of varying intensity. The intensities of the 0 and \pm 1 half-cycles at intensity (i) are labeled with red dots, and the intensities of the 0 and \pm 1 halfcycles at intensity (iii) are shown with blue dots. Additionally, the slope of the emission curve near the red dots is shown with a red dashed line and near the blue dots with a blue dashed line. Note that due to the nonlinearity of the emission the slope decreases at higher intensities. This decrease in the emission rate growth means that the blue dots are closer together than the red dots. In other words, at higher intensities, the emission from the \pm 1 half-cycles catches up to the emission from half-cycle 0 . Indeed, in the inset we show the ratio of the charge emitted from one of the \pm 1 half- 
cycles to that emitted from half-cycle 0 as $Q_{1} / Q_{0}$, and we see that this ratio increases from intensity (i) to (iii). This nonlinear, "catching up" behavior underlies the shift in $I_{\text {tot }}\left(\varphi_{\text {cep }}\right)$ from intensity (i) to (iii) and the vanishing point behavior.

Figs. 3c,j,k,l parallel Figs. 3b,g,h,i while considering emission from half-cycles -4 to +4 . Interestingly, in Fig. 3c, when more half-cycles are included, it is possible to observe multiple vanishing points. Although the origins of the vanishing points in Fig. $3 \mathrm{c}$ are not as readily apparent as in Fig. 3b, close inspection reveals that the vanishing point labeled (ii) in Fig. 3c results from the emitted current from the \pm 4 half-cycles catching up to that from the \pm 3 half-cycles. Labeling the charge emitted from half-cycle $\pm n$ as $Q_{n}$, we find that at intensity (i) in Fig. 3c, $Q_{0}+2 Q_{2}<$ $2 Q_{1}+2 Q_{3}$ and $Q_{0}+2 Q_{2}+2 Q_{4}<2 Q_{1}+2 Q_{3}$ while at intensity (iii) in Fig. $3 \mathrm{c}, Q_{0}+2 Q_{2}<$ $2 Q_{1}+2 Q_{3}$ and $Q_{0}+2 Q_{2}+2 Q_{4}>2 Q_{1}+2 Q_{3}$. In other words, due to the reduction in emission rate growth from the \pm 3 half-cycles, there is a relative increase in the emission contribution from the \pm 4 half-cycles that enables the cumulative emission from the even half-cycles to overtake that of the odds. (The factor of two in front of $Q_{n}$ for $n \neq 0$ derives from the symmetric pairs of halfcycles about half-cycle 0 .) The vanishing point at the lower intensity similarly arises from the emitted current from the \pm 3 half-cycles catching up to that from the \pm 2 half-cycles.

In line with the preceding discussion, as successive half-cycles are included, there continues to be competition between even and odd half-cycle contributions to the CEP-sensitive photocurrent, until the optical field strength, and hence the emitted current, of the higher-numbered half-cycle regions weakens significantly, at which point $I_{1}$ converges (i.e. $I_{1}$ stops changing significantly as more half-cycles are included in the calculation). For the intensity range shown and the pulse duration used in the model, $I_{1}$ converges after summing over half-cycles -4 to +4 (see Fig. 3c,j,k,1). 
At each vanishing point in Fig. 3a,b,c, the phase jumps instantaneously between 0 and $\pm \pi$. However, in the experimental and simulation results in Fig. 2, which use realistic pulses with residual chirp and plasmonic damping, the phase response near the vanishing point is smoother with a slightly downward slope. Initial modeling has indicated that the slope of $\angle I_{1}$ near the vanishing point is a function of the chirp and asymmetry in the pulse shape. By examining the CEP-sensitive response in detail, including the slope of $\angle I_{1}$ and the precise location and depth of the dip in $\left|I_{1}\right|$, one can likely obtain a great deal of information about the time-domain profile of the optical waveform at the surface of the emitter. (See sensitivity of vanishing points to changes in pulse dispersion in Supplementary Information Fig. 9 and the Supplementary Information Section VIII.) Indeed, the sensitivity of the dips and phase shifts to subtle changes in the optical electric field allowed us to confirm the field enhancement at the apices of the nanoantennas, and to refine our characterization of the damping time of the nanoantennas' plasmonic response.

The appearance of vanishing points in the CEP-sensitive current response is a general phenomenon that should be observable for any system where the current is field driven with similar nonlinear characteristics to that of FN tunneling (see Supplementary Information Section VII). Further experimental and theoretical analysis of vanishing point behaviors have the potential to probe the physics of strong-field electron emission dynamics in solids as well as gases, to better characterize the precise waveforms driving optical-field photoemission, and to sense subtle changes in the electronic structure of surfaces.

\section{References}

1. Putnam, W. P., Hobbs, R. G., Keathley, P. D., Berggren, K. K. \& Kärtner, F. X. Opticalfield-controlled photoemission from plasmonic nanoparticles. Nat. Phys. 13, 335-339 (2017). 2. Rybka, T. et al. Sub-cycle optical phase control of nanotunnelling in the single-electron regime. Nat. Photonics 10, 667-670 (2016). 
3. Kruger, M., Schenk, M. \& Hommelhoff, P. Attosecond control of electrons emitted from a nanoscale metal tip. Nature 475, 78-81 (2011).

4. Herink, G., Solli, D. R., Gulde, M. \& Ropers, C. Field-driven photoemission from nanostructures quenches the quiver motion. Nature 483, 190-193 (2012).

5. Schenk, M., Krüger, M. \& Hommelhoff, P. Strong-Field Above-Threshold Photoemission from Sharp Metal Tips. Phys. Rev. Lett. 105, 257601 (2010).

6. Paasch-Colberg, T. et al. Solid-state light-phase detector. Nat. Photonics 8, 214-218 (2014).

7. Ahn, B. et al. Attosecond-controlled photoemission from metal nanowire tips in the fewelectron regime. APL Photonics 2, 036104 (2017).

8. Swanwick, M. E. et al. Nanostructured Ultrafast Silicon-Tip Optical Field-Emitter Arrays. Nano Lett. 14, 5035-5043 (2014).

9. Dombi, P. et al. Ultrafast Strong-Field Photoemission from Plasmonic Nanoparticles. Nano Lett. 13, 674-678 (2013).

10. Rácz, P. et al. Measurement of Nanoplasmonic Field Enhancement with Ultrafast Photoemission. Nano Lett. 17, 1181-1186 (2017).

11. Piglosiewicz, B. et al. Carrier-envelope phase effects on the strong-field photoemission of electrons from metallic nanostructures. Nat. Photonics 8, 37-42 (2014).

12. Paulus, G. G. et al. Absolute-phase phenomena in photoionization with few-cycle laser pulses. Nature 414, 182-184 (2001).

13. Kling, M. F. et al. Imaging of carrier-envelope phase effects in above-threshold ionization with intense few-cycle laser fields. New J. Phys. 10, 025024 (2008).

14. Apolonski, A. et al. Observation of Light-Phase-Sensitive Photoemission from a Metal. Phys. Rev. Lett. 92, 073902 (2004).

15. Dombi, P. et al. Direct measurement and analysis of the carrier-envelope phase in light pulses approaching the single-cycle regime. New J. Phys. 6, 39 (2004).

16. Rathje, T. et al. Review of attosecond resolved measurement and control via carrierenvelope phase tagging with above-threshold ionization. J. Phys. B At. Mol. Opt. Phys. 45, 074003 (2012).

17. Peng, L.-Y., Pronin, E. A. \& Starace, A. F. Attosecond pulse carrier-envelope phase effects on ionized electron momentum and energy distributions: roles of frequency, intensity and an additional IR pulse. New J. Phys. 10, 025030 (2008).

18. Hobbs, R. G. et al. Mapping Photoemission and Hot-Electron Emission from Plasmonic Nanoantennas. Nano Lett. (2017). doi:10.1021/acs.nanolett.7b02495

19. Yalunin, S. V., Gulde, M. \& Ropers, C. Strong-field photoemission from surfaces: Theoretical approaches. Phys. Rev. B 84, 195426 (2011).

20. Bormann, R., Gulde, M., Weismann, A., Yalunin, S. V. \& Ropers, C. Tip-Enhanced Strong-Field Photoemission. Phys. Rev. Lett. 105, 147601 (2010). 
21. Kusa, F., Echternkamp, K. E., Herink, G., Ropers, C. \& Ashihara, S. Optical field emission from resonant gold nanorods driven by femtosecond mid-infrared pulses. AIP Adv. 5, 077138 (2015).

22. Keldysh, L. V. Ionization in the Field of a Strong Electromagnetic Wave. Sov Phys JETP 20, 1307-1314 (1965).

\section{$\underline{\text { Acknowledgements }}$}

We would like to thank Jim Daley for his assistance in device fabrication. This work was supported by the United States Air Force Office of Scientific Research (AFOSR) through grants FA9550-12-1-0499 and FA9550-19-1-0065, the Center for Free-Electron Laser Science at DESY, the European Research Council under the European Union's Seventh Framework Programme (FP7/2007-2013) through the Synergy Grant 'Frontiers in Attosecond X-ray Science: Imaging and Spectroscopy' (AXSIS) (609920), and The Hamburg Center for Ultrafast Imaging: Structure, Dynamics and Control of Matter at the Atomic Scale, an excellence cluster of the Deutsche Forschungsgemeinschaft. R.G.H. acknowledges support for the device fabrication work from the Center for Excitonics, an Energy Frontier Research Center funded by the US Department of Energy, Office of Science, Office of Basic Energy Sciences under award number DE-SC0001088 and support provided by the Royal Society-Science Foundation Ireland University Research Fellowship.

\section{$\underline{\text { Author Contributions }}$}

P.D.K. and W.P.P. conceived of the experiment and contributed equally to this work. W.P.P., R.G.H., and Y.Y. fabricated the devices. P.D.K., W.P.P., and P.V. performed the measurements, collected the experimental data, and performed the numerical analysis. P.D.K., W.P.P., and P.V. composed the manuscript. P.D.K., W.P.P., P.V., R.G.H., Y.Y., K.K.B., and F.X.K. interpreted the results and contributed to the final manuscript. 


\section{Competing Financial Interests}

The authors declare no competing financial interests.

\section{$\underline{\text { Methods }}$}

Few-cycle optical source. A supercontinuum-based fiber laser source at a repetition rate of $\approx$ $78.4 \mathrm{MHz}$, central wavelength of $\approx 1177 \mathrm{~nm}$ and a duration of $\approx 10 \mathrm{fs}$, that is $\approx 2.5$ optical cycles, full-width-at-half-maximum (FWHM) was used. For the experimental characterization of $I_{1}$, the carrier-envelope-offset frequency $f_{\text {ceo }}$ of our incident pulse train was locked to a stable reference

at $100 \mathrm{~Hz}$. In this locked state, the $n^{\text {th }}$ pulse in the train had a CEP phase of $\varphi_{\text {cep }}[n]=2 \pi n \frac{f_{\text {ceo }}}{f_{\text {rep }}}+$ $\varphi_{0}$, where $f_{\text {rep }}$ is the repetition rate of the incident pulse train and $\varphi_{0}$ is an absolute phase offset. We have characterized the CEP noise of our system to be $\approx 157 \mathrm{mrad}$ root-mean-square (rms) when integrated from $5 \mathrm{mHz}$ to $5 \mathrm{MHz}^{25}$. For further details regarding the optical source and phase stabilization methods used in this work, please refer to Ref. ${ }^{25}$.

Nanoantenna layout and fabrication. The nano-triangles were arranged in a square grid $(20 \mu \mathrm{m} \times 20 \mu \mathrm{m})$ with a nominal spacing of $480 \mathrm{~nm}$. Each nano-triangle in the array is nominally isosceles in shape, with a target base width of $180 \mathrm{~nm}$, altitude of $240 \mathrm{~nm}$, and thickness of $20 \mathrm{~nm}$. The triangular shape of the antennas was chosen to break the system's inversion symmetry, thereby increasing the CEP-sensitivity of the emitted photocurrent when driven into the regime of opticalfield photoemission ${ }^{1}$. Each nano-triangle has a radius of curvature (ROC) at its apex of $\approx 10 \mathrm{~nm}$.

The nano-triangle array and their supporting electrodes were fabricated in two major steps. Firstly, the nano-triangle array was patterned onto an ITO-coated sapphire substrate via electron beam lithography. Secondly, the ITO-coated substrate was patterned via optical lithography, and 
the ITO in designated areas was etched away to create the emitter and collector electrode structures. The details of each fabrication step are thoroughly described in the Supplementary Information of Ref. ${ }^{1}$.

Quasi-static tunneling models. In our FN quasi-static tunneling model, we express the field at the tip surface in the form $F(t)=\mathfrak{R}\left\{F_{0} \xi(t) \exp \left[j\left(\omega_{0} t+\varphi_{\text {cep }}\right)\right]\right\}$, where $F_{0}$ is the peak electric field strength, $\xi(t)$ is the normalized complex amplitude envelope, $\omega_{0}$ is the center frequency of the incident optical pulse, $\varphi_{\text {cep }}$ is the carrier-envelope-phase, and $j$ is the imaginary constant. For cases where plasmonic reshaping of the input pulse was taken into account, such as for modeling the experimental data in Fig. 2, we used the relation

$$
\xi(t) \propto \mathcal{F}^{-1}\left\{\mathcal{F}\left\{\xi_{0}(t)\right\} g(\omega)\right\}
$$

where $\mathcal{F}$ denotes a Fourier transform, $\xi_{0}(t)$ is the normalized complex amplitude envelope of the incident pulse, and $g(\omega)$ is the transfer-function due to the plasmonic nanostructure. We approximated the plasmonic nanostructure as a damped harmonic oscillator ${ }^{1,23,24}$, and took

$$
g(\omega) \propto \frac{1}{\omega_{\text {res }}^{2}-\left(\omega+\omega_{0}\right)^{2}-j\left(\omega+\omega_{0}\right) / \tau}
$$

where $\omega_{\text {res }}=2 \pi c / \lambda_{\text {res }}$ is the resonance frequency of the plasmonic nanoantenna, and $c$ the speed of light in vacuum. (The damped harmonic oscillator model is further motivated in the Supplementary Information of Ref. ${ }^{1}$.)

The field $F(t)$ modulates the surface potential barrier, and if sufficiently strong, it can pull the barrier down so far as to enable significant electron tunneling from the nanoantenna and into the surrounding air or vacuum before reversing polarity. We used the FN expression

$$
I_{\mathrm{FN}}(t) \propto F(t)^{2} \Theta(-F(t)) \exp \left\{\frac{4 \sqrt{2 m \phi^{3}}}{3 \hbar e F(t)}\right\}
$$


to account for the quasi-static tunneling rate, where $\Theta(x)$ is the Heaviside step function (this function ensures we only consider emission when $F(t)<0), m$ is the electron mass, $\phi$ the material work function, $\hbar$ the normalized Planck's constant, and $e$ the electron charge. We integrated $I_{\mathrm{FN}}$ over the entire pulse duration to determine the emitted charge per pulse $Q$, which is related to the integrated total photoemission current by the repetition rate of the laser: $I_{\text {tot }}=Q f_{\text {rep }}$.

To arrive at the model results presented in Fig. 2, we started with a $\cos ^{2}$-shaped optical pulse. This model pulse derived from a fit to the time-domain measurement of the experimental pulse that was characterized via two-dimensional spectral shearing interferometry $(2 \mathrm{DSI})^{25,26}$. The expected field profile at the nanoantennas' apices was then approximated by filtering this model pulse with the harmonic oscillator resonator response $g(\omega)$ and setting $F_{0}=F_{\text {inc }} \times \mathrm{FE}$, where $F_{\text {inc }}$ is the peak incident field strength of the laser pulse, and FE the field-enhancement factor of the nanoantenna.

The field-enhancement factor FE was approximated by fitting the quasi-static FN model of the total average photocurrent $I_{0}$ to measurements of $I_{0}$ over a range of incident intensities in the optical-field emission regime ${ }^{1,21}$. Using the measured spot size and pulse energy, $F_{\text {inc }}$ was calculated. The following iterative process was then used to fit FE: (1) the FN model was used for the entire dataset to obtain a first estimate for the field enhancement; (2) only those enhanced intensity values such that $\gamma<1$ were used to obtain the next field enhancement factor; (3) step (2) was repeated until the process converged to the final field enhancement factor value ${ }^{1}$. In Supplementary Information Fig. 4, we show the fit results for a single scan. In Supplementary Information Table 1, we compile field enhancement factors from nine separate scans for reference. The resultant FE fits ranged from $28.9 \times$ to $35.5 \times$ with an average FE of $(32.6 \pm 2.4) \times$ (see Supplementary Information Table 1, Supplementary Information Fig. 4, and Supplementary 
Information Section V). For the model results shown in Fig. 2, FE was taken to be $32.6 \times$ to match the average measured field enhancement.

The damping time $\tau$ and the resonant wavelength $\lambda_{\text {res }}$ followed from fits to measurements of the nanoantennas' extinction spectra (see Supplementary Information Fig. 3 and Supplementary Information Section IV). Fits to the nanoantennas' extinction spectra between 700-1400 nm yielded $\lambda_{\text {res }} \approx 1105 \mathrm{~nm}$ and $\tau$ values ranging from 6.4 to 6.9 fs (see Supplementary Information Fig. 3 and further discussion in Supplementary Information Section IV). The final value of $\tau=$ $6.8 \mathrm{fs}$ used to create the model results in Fig. 2 was chosen as it provided the best fit to the experimental data.

To account for the fact that each nano-triangle was exposed to a different peak intensity due to tight-focusing, we integrated the calculated electron emission from an array of nano-triangle apices over the focal spot of the incident beam. Using this integrated emission, we calculated the expected CEP-sensitivity, i.e. $I_{1} / I_{0}$. Multiplying this sensitivity by our measurement of $I_{0}$, we found the CEP-dependent current shown by the dashed blue line in Fig. 2. We note that while recent results show that tightly-focused, broadband optical pulses exhibit strong phase shifts in excess of the predicted Guoy phase near the region of the focus ${ }^{27}$, this effect can be safely ignored here as the emitters are only $20 \mathrm{~nm}$ tall and were located near the minimum beam waist where the CEP does not exhibit transverse dependence.

Measurements of CEP-sensitive photocurrent. The way in which the CEP-sensitive current was detected and analyzed in this work using a slowly oscillating $\varphi_{\text {cep }}$ and lock-in detection of $I_{1}$ was of critical importance. Photocurrents were first amplified and converted to a voltage signal using a transimpedance amplifier. Using lock-in detection, we measured the magnitude and phase of 
this amplified voltage signal oscillating at $f_{\text {ceo }}$; this magnitude and phase corresponds to $\left|I_{1}\right|$ and $\angle I_{1}$ respectively. We verified that the measured $I_{1}$ signal was indeed controlled by the CEP of the incident pulse by translating a barium-fluoride wedge through the beam ${ }^{1}$ and verifying the expected phase shift imparted on $\angle I_{1}$ as a function of the wedge insertion (see results in Supplementary Information Fig. 1 and Supplementary Information Section I).

A variable neutral density filter was then used to alter the incident pulse energy while monitoring $I_{1}$. We note that during the intensity scans we observed a fluctuation in measurements of $\left|I_{1}\right|$ of less than $20 \% \mathrm{rms}$, a fluctuation of $\angle I_{1}$ of less than $180 \mathrm{mrad} \mathrm{rms}$, and a noise floor for the current measurements of around 20-40 fA. Additionally, the magnitude of the CEP sensitivity (away from the vanishing point) was on the order of $5 \times 10^{-5}$.

\section{Data Availability}

The data that support the plots within this paper and other findings of this study are included in this published article (and its Supplementary Information file) and are available from the corresponding authors upon reasonable request.

\section{$\underline{\text { References }}$}

23. Hentschel, M., Utikal, T., Giessen, H. \& Lippitz, M. Quantitative Modeling of the Third Harmonic Emission Spectrum of Plasmonic Nanoantennas. (2012). doi:10.1021/n1301686x

24. Metzger, B., Hentschel, M., Lippitz, M. \& Giessen, H. Third-harmonic spectroscopy and modeling of the nonlinear response of plasmonic nanoantennas. Opt. Lett. 37, 4741-4743 (2012).

25. Putnam, W. P. et al. Few-cycle, carrier-envelope-phase-stable laser pulses from a compact supercontinuum source. JOSA B 36, A93-A97 (2019).

26. Birge, J. R., Ell, R. \& Kärtner, F. X. Two-dimensional spectral shearing interferometry for few-cycle pulse characterization. Opt. Lett. 31, 2063-2065 (2006).

27. Hoff, D. et al. Tracing the phase of focused broadband laser pulses. Nat. Phys. 13, 947951 (2017). 